\title{
Estimates of Invariant Metrics on Pseudoconvex Domains of Finite Type in $\mathbb{C}^{3}$
}

\author{
Sanghyun Cho' and Young Hwan You \\ ${ }^{1}$ Department of Mathematics, Sogang University, Seoul 121-742, Republic of Korea \\ ${ }^{2}$ Department of Mathematics, Indiana University East, Richmond, IN 47374, USA \\ Correspondence should be addressed to Sanghyun Cho; shcho@sogang.ac.kr
}

Received 30 June 2014; Accepted 6 October 2014; Published 12 November 2014

Academic Editor: Sung G. Kim

Copyright (c) 2014 S. Cho and Y. H. You. This is an open access article distributed under the Creative Commons Attribution License, which permits unrestricted use, distribution, and reproduction in any medium, provided the original work is properly cited.

\begin{abstract}
Let $\Omega$ be a smoothly bounded pseudoconvex domain in $\mathbb{C}^{3}$ and assume that $z_{0} \in b \Omega$ is a point of finite 1-type in the sense of D'Angelo. Then, there are an admissible curve $\Gamma \subset \Omega \cup\left\{z_{0}\right\}$, connecting points $q_{0} \in \Omega$ and $z_{0} \in b \Omega$, and a quantity $M(z, X)$, along $z \in \Gamma$, which bounds from above and below the Bergman, Caratheodory, and Kobayashi metrics in a small constant and large constant sense.
\end{abstract}

\section{Introduction}

Let $\Omega$ be a smoothly bounded domain in $\mathbb{C}^{n}$ and let $X$ be a holomorphic tangent vector at a point $z$ in $\Omega$, and let us denote the Bergman, Caratheodory, and Kobayashi metrics at $z$ by $B_{\Omega}(z ; X), C_{\Omega}(z ; X)$, and $K_{\Omega}(z ; X)$, respectively. When $\Omega$ is a strongly pseudoconvex domain in $\mathbb{C}^{n}$, the optimal boundary behavior of the above metrics is well understood. For weakly pseudoconvex domains of finite type in $\mathbb{C}^{n}$, several authors found some results about these metrics. But in each case, the lower bounds are different from the upper bounds [1-5]. In [6], Catlin got optimal estimates in a small constant and large constant sense for pseudoconvex domains of finite type in $\mathbb{C}^{2}$. For pseudoconvex domains of finite type in $\mathbb{C}^{n}$, the first author and Herbort extended Catlin's result to the case that the Levi-form at $z_{0}$ has corank one $[7,8]$ or homogeneous finite diagonal type near $z_{0} \in b \Omega[9,10]$.

To estimate the above invariant metrics, we need a complete geometric analysis near $z_{0} \in b \Omega$ of finite type, and then we construct a family of plurisubharmonic functions with maximal Hessian near $b \Omega$. However, this construction is really technical and known only for special types of domains mentioned above, but not for arbitrary pseudoconvex domains of finite type in $\mathbb{C}^{n}$, even for $n=3$ case. Meanwhile, it is useful to understand the behavior of a holomorphic function near $z_{0} \in b \Omega$ if we have precise estimates of the invariant metric along some curves.

In the sequel, we let $\Omega$ be a smoothly bounded pseudoconvex domain in $\mathbb{C}^{3}$ with smooth defining function $r$ and let $z_{0} \in b \Omega$. Let $\mathscr{M}\left(z_{0}\right)=\left(1, m, m_{3}\right)$ be Catlin's multitype [11]. Thus, $m=T_{\mathrm{BG}}\left(z_{0}\right)$ is the type in the sense of "BloomGraham." If $m_{3}=\Delta_{1}\left(z_{0}\right)$, then $\Omega$ is an $h$-extensible domain [12] and Herbort [10] got an estimate in this case. Here, $\Delta_{q}\left(z_{0}\right)$ denotes finite $q$-type in the sense of D'Angelo. Thus, we assume that $m \leq m_{3}<\Delta_{1}\left(z_{0}\right)$. Regular finite 1-type at $z_{0} \in b \Omega$ is the maximum order of vanishing of $r \circ \gamma$ for all one complex dimensional regular curves $\gamma, \gamma(0)=z_{0}$ and $\gamma^{\prime}(0) \neq 0$. We denote the regular finite 1-type at $z_{0}$ by $T_{\Omega}^{\mathrm{reg}}\left(z_{0}\right)$. Note that $T_{\Omega}^{\text {reg }}\left(z_{0}\right)$ is a positive integer and $T_{\Omega}^{\text {reg }}\left(z_{0}\right) \leq \Delta_{1}\left(z_{0}\right)$.

Assuming that $T_{\Omega}^{\mathrm{reg}}\left(z_{0}\right)=\eta<\infty$, there exist coordinate functions $z=\left(z_{1}, z_{2}, z_{3}\right)$ defined in a neighborhood $V$ of $z_{0}$ such that $z_{0}=0$ and $\left|\partial r / \partial z_{3}\right| \geq c_{0}$ on $V$ for a uniform constant $c_{0}>0$, and $\left|r\left(z_{1}, 0,0\right)\right|$ vanishes to order $\eta$, and $\left(\partial r / \partial z_{2}\right)(0)=$ 0 (Theorem 2.1 in [13]). With these coordinates at hand, set

$$
\begin{aligned}
L_{k} & =\frac{\partial}{\partial z_{k}}-\left(\frac{\partial r}{\partial z_{3}}\right)^{-1} \frac{\partial r}{\partial z_{k}} \frac{\partial}{\partial z_{3}}:=\frac{\partial}{\partial z_{k}}+e_{k}(z) \frac{\partial}{\partial z_{3}}, \\
k & =1,2, \\
L_{3} & =\frac{\partial}{\partial z_{3}} .
\end{aligned}
$$


Then, $e_{k}(0)=0, k=1,2$, and $\left\{L_{1}, L_{2}, L_{3}\right\}$ form a basis of $\mathbb{C} T^{(1,0)}(V)$ provided $V$ is sufficiently small. For any integer $j$, $k>0$, set

$$
\mathscr{L}_{j, k} \partial \bar{\partial} r(z)=\underbrace{L_{2} \cdots L_{2}}_{(j-1) \text { times }} \underbrace{\bar{L}_{2} \cdots \bar{L}_{2}}_{(k-1) \text { times }} \partial \bar{\partial} r(z)\left(L_{2}, \bar{L}_{2}\right)(z)
$$

and define

$$
C_{l}(z)=\max \left\{\left|\mathscr{L}_{j, k} \partial \bar{\partial} r(z)\right|: j+k=l\right\} .
$$

Let $X=a_{1} L_{1}+a_{2} L_{2}+a_{3} L_{3}$ be a holomorphic tangent vector at $z \in \Omega$ and set

$$
\begin{aligned}
M(z ; X)= & \left|a_{1}\right||r(z)|^{-1 / \eta}+\left|a_{2}\right| \sum_{l=2}^{m}\left(\frac{C_{l}(z)}{|r(z)|}\right)^{1 / l} \\
& +\left|a_{3}\right||r(z)|^{-1} .
\end{aligned}
$$

Let $\Gamma \subset \Omega \cup\left\{z_{0}\right\}$ be the admissible curve defined in (20). Our main result is as follows.

Theorem 1. Let $\Omega \subset \subset \mathbb{C}^{3}$ be a smoothly bounded pseudoconvex domain and assume $z_{0} \in b \Omega$ is a point of finite 1-type in the sense of D'Angelo; that is, $\Delta_{1}\left(z_{0}\right)<\infty$. Then, there exist a neighborhood $V$ about $z_{0}$, an admissible curve $\Gamma \subset \Omega \cup\left\{z_{0}\right\}$ connecting $q_{0} \in \Omega$ and $z_{0}$, and positive constants $c$ and $C$ such that, for all $X=a_{1} L_{1}+a_{2} L_{2}+a_{3} L_{3}$ at $z \in V \cap \Gamma \cap \Omega$,

$$
\begin{gathered}
c M(z ; X) \leq B_{\Omega}(z ; X) \leq C M(z ; X) \\
c M(z ; X) \leq C_{\Omega}(z ; X) \leq C M(z ; X) \\
c M(z ; X) \leq K_{\Omega}(z ; X) \leq C M(z ; X) .
\end{gathered}
$$

To prove Theorem 1, we use special coordinates constructed in Section 2 of [13]. Thus, there is a special direction $d,|d|=1$, so that, for each $\delta>0$, the two-dimensional slice $D_{\delta}:=\left\{\left(z_{2}, z_{3}\right) ; r\left(d \delta^{1 / \eta}, z_{2}, z_{3}\right)<0\right\}$ becomes a pseudoconvex domain of finite type in $\mathbb{C}^{2}$, whose type is less than or equal to $m=T_{\mathrm{BG}}\left(z_{0}\right)$. We then apply the method which holds for the domains of finite type in $\mathbb{C}^{2}$ as in [6]. To avoid the difficulty to push out the domain in $z_{1}$-direction, we use a bumping theorem of Cho [14].

\section{Special Coordinates}

Let $\Omega \subset \mathbb{C}^{3}$ and $z_{0} \in b \Omega$ be as in Section 1 . We may assume that $z_{0}=0$. In this section, we consider special coordinates defined near $z_{0} \in b \Omega$ and then construct "balls" which are of maximal size on which $r(z)$ changes by no more than some prescribed number $\delta>0$. In the following, we let $\alpha=\left(\alpha_{1}, \alpha_{2}\right)$ and $\beta=\left(\beta_{1}, \beta_{2}\right)$ be multi-indices with respect to $z^{\prime}=\left(z_{1}, z_{2}\right)$ variables. In Theorem 2.1 of [13], You constructed special coordinates which represent the local geometry of $b \Omega$ near $z_{0}$.

Theorem 2. Let $\Omega$ be a smoothly bounded pseudoconvex domain in $\mathbb{C}^{3}$ with smooth defining function $r$ and assume
$T_{\Omega}^{r e g}(0)=\eta<\infty, 0 \in b \Omega$. Then, there is a holomorphic coordinate system $z=\left(z_{1}, z_{2}, z_{3}\right)$ about 0 such that

$$
\begin{aligned}
r(z)=\operatorname{Re} z_{3}+ & \sum_{\substack{|\alpha|+|\beta|=m \\
|\alpha|,|\beta|>0}}^{\eta} a_{\alpha, \beta} z^{\prime \alpha} \bar{z}^{\prime \beta} \\
& +\mathcal{O}\left(\left|z_{3}\right||z|+\left|z^{\prime}\right|^{\eta+1}\right),
\end{aligned}
$$

(2) $|r(t, 0,0)| \lesssim|t|^{\eta}$,

where $z^{\prime}=\left(z_{1}, z_{2}\right)$ and where

$$
a_{\alpha, \beta} \neq 0 \text { forsome } \alpha, \beta \text { with } \alpha_{1}=\beta_{1}=0, \alpha_{2}+\beta_{2}=m \text {. }
$$

Remark 3. (1) The second condition in (6) and the property (7) say that $r(z)$ vanishes to order $\eta$ along $z_{1}$ axis and order $m$ along $z_{2}$ axis. These properties are crucial for the construction of maximal polydiscs $Q_{c \delta}\left(z^{\delta}\right)$ contained in $\Omega$.

(2) There are much more terms (mixed with $z_{1}$ and $z_{2}$ and their conjugates) in the summation part of (6) compared to the $h$-extensible domain cases.

According to Proposition 2.6 and Remark 2.7 of [13], there are pairs of integers $\left(p_{v}, q_{v}\right), v=1, \ldots, N$, such that the terms satisfying $\alpha_{1}+\beta_{1}=p_{v}$ and $\alpha_{2}+\beta_{2}=q_{v}$ with $\alpha_{2}>0$ and $\beta_{2}>0$ are dominant terms in the summation part of (6). Also, there is a small constant $a_{0}>0$ and a fixed direction $d$, $|d|=1$, in $z_{1}$ direction, such that, for each fixed $\delta>0$ and for all $z_{1}$ satisfying $\left|z_{1}-d \delta^{1 / \eta}\right|<a_{0} \delta^{1 / \eta}$, those major terms in the summation part of (6) satisfy

$$
\left|\frac{\partial^{q_{v}}}{\partial z_{2}^{\alpha_{2}} \bar{\partial} \bar{z}_{2}^{\beta_{2}}} r\left(z_{1}, 0,0\right)\right| \approx\left|z_{1}\right|^{p_{v}} \approx \delta^{p_{v} / \eta}
$$

where $\alpha_{2}+\beta_{2}=q_{v}$ and where $\alpha_{2}>0$ and $\beta_{2}>0$.

Now, let us fix $z_{1}$ with $\left|z_{1}-d \delta^{1 / \eta}\right|<a_{0} \delta^{1 / \eta}$ and consider the two-dimensional slice $D_{z_{1}}:=\left\{\left(z_{2}, z_{3}\right): r\left(z_{1}, z_{2}, z_{3}\right)<0\right\}$. For each $z=\left(z_{1}, 0, z_{3}\right)$ near $b \Omega$, set $\pi(z)=\left(z_{1}, 0, e_{\delta}\right):=$ $\tilde{z}_{1} \in b \Omega$, where $\pi(z)$ is the projection of $z$ onto $b \Omega$ along $z_{3}$ direction. On $D_{z_{1}}$, following the argument in twodimensional case as in the proof of Proposition 1.1 in [6], we construct special coordinates $\zeta=\left(\zeta_{1}, \zeta_{2}, \zeta_{3}\right)=\left(z_{1}, z_{2}, \zeta_{3}\right)$ about $\widetilde{z}_{1}$ so that, in terms of new coordinates, there are no pure terms in $z_{2}$ variable in the expression of $r(z)$ in (6).

Proposition 4. For each fixed $\widetilde{z}_{1}=\left(z_{1}, 0, e_{\delta}\right) \in V \cap b \Omega$, there exists a holomorphic coordinate system $z=\Phi_{\widetilde{z}_{1}}(\zeta)=$ $\left(z_{1}, z_{2}, \Phi_{3}(\zeta)\right), \zeta=\left(\zeta_{1}, \zeta_{2}, \zeta_{3}\right)=\left(z_{1}, z_{2}, \zeta_{3}\right)$, where $\Phi_{3}(\zeta)$ is defined by

$$
\begin{aligned}
\Phi_{3}(\zeta)= & e_{\delta}+\left(\frac{\partial r}{\partial z_{3}}\left(\widetilde{z}_{1}\right)\right)^{-1} \\
& \times\left(\frac{\zeta_{3}}{2}-\sum_{l=2}^{m} c_{l}\left(\widetilde{z}_{1}\right) \zeta_{2}^{l}-\frac{\partial r}{\partial z_{2}}\left(\widetilde{z}_{1}\right) \zeta_{2}\right) \\
:= & e_{\delta}+d_{0}\left(\widetilde{z}_{1}\right) \zeta_{3}+\sum_{l=1}^{m} d_{l}\left(\widetilde{z}_{1}\right) \zeta_{2}^{l},
\end{aligned}
$$


and the function $\rho$, given by $\rho\left(z_{1}, \zeta^{\prime \prime}\right):=r \circ \Phi_{\widetilde{z}_{1}}\left(z_{1}, \zeta^{\prime \prime}\right), \zeta^{\prime \prime}=$ $\left(\zeta_{2}, \zeta_{3}\right)$, satisfies

$$
\rho\left(z_{1}, \zeta^{\prime \prime}\right)=\operatorname{Re}\left(\Phi_{3}(\zeta)\right)+\sum_{\substack{j+k=2 \\ j, k>0}}^{m} a_{j, k}\left(\widetilde{z}_{1}\right) \zeta_{2}^{j} \zeta_{2}^{k}+E(\zeta),
$$

where

$$
E(\zeta)=\mathcal{O}\left(\left|\Phi_{3}(\zeta)\right||\zeta|+\sum_{\nu=1}^{N}\left|z_{1}\right|^{1+p_{v}}\left|\zeta_{2}\right|^{q_{\nu}}+\left|\zeta_{2}\right|^{m+1}\right) .
$$

In view of (6) and (8), the major terms in (10) are $a_{j, k}\left(\widetilde{z}_{1}\right) \zeta_{2}^{j} \bar{\zeta}_{2}^{k}$ where $j+k=\alpha_{2}+\beta_{2}=q_{v}$ for some $\alpha_{2}$ and $\beta_{2}$ with $\alpha_{2}>0$ and $\beta_{2}>0$. Also, from (8), it follows that

$$
\left|a_{j, k}\left(\widetilde{z}_{1}\right) \zeta_{2}^{j} \bar{\zeta}_{2}^{k}\right| \approx\left|z_{1}\right|^{p_{v}}\left|z_{2}\right|^{q_{v}}
$$

and these terms control the error terms $\left|z_{1}\right|^{1+p_{\nu}}\left|\zeta_{2}\right|^{q_{\nu}}$ in $E(\zeta)$. As in Section 1 in [6], set

$$
A_{l}\left(\widetilde{z}_{1}\right)=\max \left\{\left|a_{j, k}\left(\widetilde{z}_{1}\right)\right| ; j+k=l\right\}, \quad l=2, \ldots, m,
$$

and for each sufficiently small $\delta>0$, we set

$$
\tau\left(\widetilde{z}_{1}, \delta\right)=\min \left\{\left(\frac{\delta}{A_{l}\left(\widetilde{z}_{1}\right)}\right)^{1 / l} ; 2 \leq l \leq m\right\} .
$$

Thus, for all $z_{1}$ with $\left|z_{1}-d \delta^{1 / \eta}\right|<a_{0} \delta^{1 / \eta}$, it follows from (8) and (14) that

$$
\tau\left(\widetilde{z}_{1}, \delta\right) \lesssim\left(\frac{\delta}{\left|z_{1}\right|^{p_{\nu}}}\right)^{1 / q_{\nu}}, \quad v=1, \ldots, N,
$$

and hence the summation part of (10) is dominated by $C \delta$.

For each $\widetilde{z}=\left(z_{1}, 0, z_{3}\right)$ near $b \Omega$, set $\widetilde{\zeta}=\Phi_{\widetilde{z}_{1}}^{-1}(\widetilde{z})=$ $\left(z_{1}, 0, \widetilde{\zeta}_{3}\right)$, where $\Phi_{\widetilde{z}_{1}}$ is the function defined in Proposition 4. For each small $e>0$, set

$$
\begin{aligned}
R_{e \delta}(\widetilde{\zeta})= & \left\{\zeta:\left|\zeta_{1}-z_{1}\right|<e \delta^{1 / \eta},\left|\zeta_{2}\right|<e \tau\left(\widetilde{z}_{1}, \delta\right),\right. \\
& \left.\left|\zeta_{3}-\widetilde{\zeta}_{3}\right|<e \delta\right\}, \\
Q_{e \delta}(\widetilde{z})= & \left\{z: z=\Phi_{\widetilde{z}_{1}}(\zeta), \zeta \in R_{e \delta}(\widetilde{\zeta})\right\} .
\end{aligned}
$$

For each $\sigma>0$, let $\Omega_{\sigma}=\{z ; r(z)<\sigma\}$ and define

$$
\begin{gathered}
S(\sigma)=\{z \in V:-\sigma<r(z) \leq \sigma\} \\
S^{-}(\sigma)=\{z \in V:-\sigma<r(z) \leq 0\},
\end{gathered}
$$

and set $\widetilde{z}^{\delta}=\left(d \delta^{1 / \eta}, 0, e_{\delta}\right) \in b \Omega$, where $z_{1}$ is replaced by $d \delta^{1 / \eta}$ in $\widetilde{z}_{1}=\left(z_{1}, 0, e_{\delta}\right)$. The following theorem is about the existence of plurisubharmonic function with maximal Hessian. In [6], for the domains in $\mathbb{C}^{2}$, Catlin constructed the functions with maximal Hessian on the strip $S(\delta) \cap V$. However, for regular finite type pseudoconvex domains in $\mathbb{C}^{3}$, we show that the functions have maximal Hessian on each ball $Q_{b \delta}\left(\widetilde{z}^{\delta}\right)$ and this will suffice to prove the boundary behavior of the invariant metrics. The proof of the following theorem can be found in Theorem 3.2 in [9].
Theorem 5. There is a small constant $b>0$ such that, for each small $\delta>0$, there is a plurisubharmonic function $g_{\delta} \in$ $C_{0}^{\infty}\left(Q_{2 b \delta}\left(\widetilde{z}^{\delta}\right)\right)$ with the following properties:

(i) $\left|g_{\delta}(\zeta)\right| \leq 1, z \in \Omega_{\delta}$,

(ii) for all $L=b_{1} L_{1}+b_{2} L_{2}+b_{3} L_{3}$ at $z$, where $z \in Q_{b \delta}\left(\widetilde{z}^{\delta}\right) \cap$ $S(b \delta)$,

$$
\partial \bar{\partial} g_{\delta}(L, \bar{L})(z) \gtrsim \delta^{-2 / \eta}\left|b_{1}\right|^{2}+\tau\left(\widetilde{z}^{\delta}, \delta\right)^{-2}\left|b_{2}\right|^{2}+\delta^{-2}\left|b_{3}\right|^{2},
$$

(iii) if $\Phi(\zeta)=\left(\zeta_{1}, \zeta_{2}, \Phi_{3}(\zeta)\right)$, where $\Phi_{3}$ is defined in (10) for $\widetilde{z}^{\delta}$, then

$$
\left|\widetilde{D}^{\alpha} g_{\delta} \circ \Phi(\zeta)\right| \leq C_{\alpha} \delta^{-\alpha_{1} / \eta} \tau\left(\widetilde{z}^{\delta}, \delta\right)^{-\alpha_{2}} \delta^{-\alpha_{3}}
$$

holds for all $\zeta \in R_{2 b \delta}\left(\widetilde{z}^{\delta}\right)$, where $\widetilde{D}^{\alpha}=\widetilde{D}_{1}^{\alpha_{1}} \widetilde{D}_{2}^{\alpha_{2}} \widetilde{D}_{3}^{\alpha_{3}}$.

Let $\Gamma \subset \Omega$ be a curve defined by

$$
\Gamma:=\left\{z^{\delta}: z^{\delta}=\left(d \delta^{1 / \eta}, 0, e_{\delta}-\frac{b \delta}{2}\right), 0 \leq \delta \leq \delta_{0}\right\},
$$

for sufficiently small $\delta_{0}>0$ and $b>0$. In the sequel, for each $z^{\delta}=\left(d \delta^{1 / \eta}, 0, e_{\delta}-b \delta / 2\right) \in \Gamma$, set $\zeta^{\delta}:=\Phi_{\widetilde{z}^{\delta}}^{-1}\left(z^{\delta}\right)$ and set $\widetilde{\Omega}=$ $\Phi_{\widetilde{z}^{\delta}}^{-1}(\Omega)$. In view of Proposition 3.4 in [9], there is a uniform small constant $c>0$ such that $R_{c \delta}\left(\zeta^{\delta}\right) \subset \subset R_{b \delta}\left(\widetilde{z}^{\delta}\right) \cap \widetilde{\Omega}$, and hence

$$
Q_{c \delta}\left(z^{\delta}\right)=\left\{z: z=\Phi_{\widetilde{z}^{\delta}}(\zeta), \zeta \in R_{c \delta}\left(\zeta^{\delta}\right)\right\} \subset \subset Q_{b \delta}\left(\widetilde{z}^{\delta}\right) \cap \Omega,
$$

provided $c>0$ and $\delta_{0}>0$ are sufficiently small. In particular, we have $\Gamma \subset \Omega \cup\left\{z_{0}\right\}$. Note that $\tau\left(z^{\delta}, \delta\right) \approx \tau\left(\widetilde{z}^{\delta}, \delta\right)$, and for $z \epsilon$ $Q_{c \delta}\left(z^{\delta}\right) \subset \Omega$, we note that $|r(z)| \approx \delta$. Thus, as in Proposition 1.3 and Corollary 1.4 in [6], we obtain that

$$
\tau\left(z^{\delta}, \delta\right)^{-1} \approx \sum_{k=2}^{m}\left(\frac{C_{k}(z)}{|r(z)|}\right)^{1 / k}, \quad z \in Q_{c \delta}\left(z^{\delta}\right),
$$

where $C_{k}(z)$ is defined in (3). In the sequel, we set $\tau_{1}=\delta^{1 / \eta}$, $\tau_{2}=\tau\left(\widetilde{z}^{\delta}, \delta\right)$, and $\tau_{3}=\delta$. If we use the plurisubharmonic weight functions constructed in Theorem 5 and follow the method to prove Theorem 6.1 in [6], we get the following estimates of the Bergman kernel along $\Gamma$.

Theorem 6. Let $z_{0} \in b \Omega$ be a point of regular finite 1-type and $T_{\Omega}^{r e g}\left(z_{0}\right)=\eta$. Then, $K_{\Omega}\left(z^{\delta}, z^{\delta}\right)$, the Bergman kernel function of $\Omega$ at $z^{\delta} \in \Gamma, \delta>0$, satisfies

$$
K_{\Omega}\left(z^{\delta}, z^{\delta}\right) \approx \delta^{-2} \tau_{1}^{-2} \tau_{2}^{-2} .
$$

\section{Metric Estimates}

In this section, we estimate the behavior of the invariant metric along $\Gamma$. In [15], Hahn got the following inequalities:

$$
C_{\Omega}(z ; X) \leq B_{\Omega}(z ; X), \quad C_{\Omega}(z ; X) \leq K_{\Omega}(z ; X) .
$$


Therefore, the estimates for the lower bounds of $C_{\Omega}(z ; X)$ will suffice for the lower bounds of $B_{\Omega}(z ; X)$ and $K_{\Omega}(z ; X)$. First, we recall the following bumping theorem [14].

Theorem 7 (Theorem 2.3 in [14]). Let $z_{0}$ be a point of finite 1-type in the boundary of a pseudoconvex domain $\Omega \subset \mathbb{C}^{n}$, defined by $\Omega=\{z: r(z)<0\}$. Then, there exist $V \ni z_{0}$ and $a$ smooth 1-parameter family of pseudoconvex domains $\Omega_{t}, 0 \leq$ $t<t_{0}$, each defined by $\Omega_{t}=\{z ; r(z, t)<0\}$, where $r(z, t)$ has the following properties:

(1) $r(z, t)$ is smooth in $z$ for $z$ near $b \Omega$ and in $t$ for $0 \leq t<$ $t_{0}$

(2) $r(z, t)=r(z)$, for $z \notin V$;

(3) $(\partial r / \partial t)(z, t) \leq 0$;

(4) $r(z, 0)=r(z)$;

(5) for $z$ in $V, \partial r / \partial t<0$.

Proof of Theorem 1. In the sequel, let us fix $\delta>0$ and, for each $z^{\delta} \in \Gamma$, set $\pi\left(z^{\delta}\right)=\widetilde{z}^{\delta}=\left(d \delta^{1 / \eta}, 0, e_{\delta}\right) \in b \Omega$ and consider the special coordinates $\zeta=\left(z_{1}, z_{2}, \zeta_{3}\right)$ and $\Phi_{\widetilde{z}^{\delta}}(\zeta)=$ $\left(z_{1}, z_{2}, \Phi_{3}(\zeta)\right)=z$, where $\Phi_{3}$ is defined in Proposition 4 . From (9), we see that $\zeta^{\delta}=\left(d \delta^{1 / \eta}, 0,-b \delta / 2 d_{0}\left(\widetilde{z}^{\delta}\right)\right):=$ $\left(\widetilde{\zeta}_{1}, \widetilde{\zeta}_{2}, \widetilde{\zeta}_{3}\right)$. We will estimate the metrics at $\zeta^{\delta}$. For all small $\delta>0$ and for each $\zeta^{\prime \prime}=\left(\zeta_{2}, \zeta_{3}\right)$, define

$$
J_{\delta}\left(\zeta^{\prime \prime}\right)=\left(\delta^{2}+\left|\zeta_{3}\right|^{2}+\sum_{k=2}^{m}\left(A_{k}\left(\widetilde{z}^{\delta}\right)\right)^{2}\left|\zeta_{2}\right|^{2 k}\right)^{1 / 2}
$$

where $A_{k}\left(\widetilde{z}^{\delta}\right)$ is defined in (13) with $\widetilde{z}_{1}$ replaced by $\widetilde{z}^{\delta}$. Let $c>0$ be the fixed constant determined in (21). Note that $\Phi_{\widetilde{z}^{\delta}}\left(d \delta^{1 / \eta}, 0,0\right)=\widetilde{z}^{\delta}$. Set

$$
\begin{gathered}
\widetilde{\Omega}_{a, \delta}=\left\{\zeta ;\left|\zeta_{1}-d \delta^{1 / \eta}\right|<c \delta^{1 / \eta},\left|\zeta_{2}\right|<a,\left|\zeta_{3}\right|<a,\right. \\
\left.\rho\left(\zeta_{1}, \zeta_{2}, \zeta_{3}\right)<0\right\}
\end{gathered}
$$

and, for each $\epsilon>0$, define

$$
\begin{gathered}
\widetilde{\Omega}_{a, \delta}^{\epsilon}=\left\{\zeta ;\left|\zeta_{1}-d \delta^{1 / \eta}\right|<c \delta^{1 / \eta},\left|\zeta_{2}\right|<a,\left|\zeta_{3}\right|<a,\right. \\
\left.\rho\left(d \delta^{1 / \eta}, \zeta^{\prime \prime}\right)<\epsilon J_{\delta}\left(\zeta^{\prime \prime}\right)\right\}
\end{gathered}
$$

and for all small $e>0$ set $B_{e}=R_{e \delta}\left(\zeta^{\delta}\right)$. By (21), we see that $\zeta^{\delta} \in B_{e} \subset \widetilde{\Omega}$ for all $e \leq c$. Note that the domains $\widetilde{\Omega}_{a, \delta}^{\epsilon}$ are pushed out only in $\zeta_{2}$ and $\zeta_{3}$ directions but not in $\zeta_{1}$ direction. To avoid the difficulty to push out $\widetilde{\Omega}$ in $\zeta_{1}$ direction, we use a bumping family of Theorem 7 . Consider a bumping family of pseudoconvex domains $\left\{\Omega_{t}\right\}_{0 \leq t \leq t_{0}}$ with front $V$ and set $D=$ $\Omega_{t_{0}}$. For each $r>0$, let $U_{r}(z)$ be a ball of radius $r>0$ with center at $z$ and set $\widetilde{U}_{r}(\zeta)=\Phi_{\widetilde{z}^{\delta}}^{-1}\left(U_{r}\left(\Phi_{\widetilde{z}^{\delta}}(\zeta)\right)\right)$. Then, there is $r_{0}>0$ such that

$$
Q_{c \delta}\left(z^{\delta}\right) \subset \Omega_{a, \delta}^{\epsilon}=\Phi_{\widetilde{z}^{\delta}}\left(\widetilde{\Omega}_{a, \delta}^{\epsilon}\right) \subset U_{r_{0} / 4}(0) \subset U_{r_{0}}(0) \subset \subset D,
$$

for all sufficiently small $a>0, \epsilon>0$, and $\delta>0$.
In view of the proof in Section 3 of [13], we have $\widetilde{\Omega}_{a, \delta} \subset$ $\widetilde{\Omega}_{a, \delta}^{\epsilon / 2} \subset \widetilde{\Omega}_{a, \delta}^{\epsilon}$ and there is a uniformly (independent of $\delta>$ 0 ) bounded function $\tilde{f}=\tilde{f}\left(\zeta_{2}, \zeta_{3}\right)$ which is holomorphic on $\widetilde{\Omega}_{a, \delta}^{\epsilon}$ and satisfies

$$
\left|Y^{\prime \prime} \tilde{f}\left(\zeta^{\delta}\right)\right| z\left|b_{2}\right| \tau_{2}^{-1}+\left|b_{3}\right| \tau_{3}^{-1}
$$

where $Y^{\prime \prime}=b_{2}\left(\partial / \partial \zeta_{2}\right)+b_{3}\left(\partial / \partial \zeta_{3}\right)$. Here, we may assume that $\tilde{f}\left(0,-b \delta / d_{0}\left(\widetilde{z}^{\delta}\right)\right)=0$. In the sequel, we let $Y$ be a vector field given by $Y=b_{1}\left(\partial / \partial \zeta_{1}\right)+b_{2}\left(\partial / \partial \zeta_{2}\right)+b_{3}\left(\partial / \partial \zeta_{3}\right)$. If $\left|b_{1}\right| \tau_{1}^{-1} \geq$ $\left|b_{2}\right| \tau_{2}^{-1}+\left|b_{3}\right| \tau_{3}^{-1}$, then set $v_{\delta}=\tau_{1}^{-1}\left(\zeta_{1}-d \delta^{1 / \eta}\right)$. Otherwise, set $v_{\delta}=\tilde{f}\left(\zeta_{2}, \zeta_{3}\right)$. From (29), we note that

$$
\left|Y v_{\delta}\left(\zeta^{\delta}\right)\right| \gtrsim \sum_{i=1}^{3}\left|b_{i}\right| \tau_{i}^{-1}
$$

Let $\psi \in C_{0}^{\infty}(U)$, where $U$ is the unit polydisc in $\mathbb{C}^{3}$, such that $\psi(z)=1$ if $\left|z_{i}\right| \leq 1 / 2, i=1,2,3$, and set

$$
\psi_{d}(\zeta)=\psi\left(\frac{\zeta_{1}-\tilde{\zeta}_{1}}{d \tau_{1}}, \frac{\zeta_{2}}{d \tau_{2}}, \frac{\zeta_{3}-\tilde{\zeta}_{3}}{d \tau_{3}}\right)
$$

and set $\beta_{\delta}=v_{\delta} \psi_{d}$. Then, $\beta_{\delta}\left(\zeta^{\delta}\right)=0$. Since $\tilde{f}$ is bounded independent of $\delta$ (and hence independent of $\zeta^{\delta}$ ), there exists a constant $C>0$, independent of $\delta$, such that $\left|\beta_{\delta}\right| \leq C$. We want to correct $\beta_{\delta}$ so that the corrected function $f_{\delta}$ becomes a uniformly bounded holomorphic function on $\widetilde{\Omega}$ satisfying the estimate (30) with $\beta_{\delta}$ replaced by $f_{\delta}$. With bumped domain $D=\Omega_{t_{0}}$ at hand, set $\widetilde{D}=\Phi_{\widetilde{z}^{\delta}}^{-1}(D)$. On $\widetilde{D}$, instead of $\widetilde{\Omega}$, we will employ weighted estimates of $\bar{\partial}$ that is essentially a replication of the proof of Theorem 6.1 in [6].

Let $g_{\delta}$ be the weight function defined in Theorem 5 and set $\tilde{g}_{\delta}=\Phi_{\widetilde{z}^{\delta}}^{*} g_{\delta}$. By replacing $\tilde{g}_{\delta}$ by $\tilde{g}_{\delta}+|\zeta|^{2}:=\phi$, we can obviously assume that $\phi$ is strictly plurisubharmonic on $\widetilde{D}$ and $\phi\left(\zeta^{\delta}\right)=0$. In view of Theorem 5, we also have

$$
\begin{array}{r}
\partial \bar{\partial} \phi(Y, \bar{Y})(\zeta) \gtrsim \tau_{1}^{-2}\left|b_{1}\right|^{2}+\tau_{2}^{-2}\left|b_{2}\right|^{2}+\tau_{3}^{-2}\left|b_{3}\right|^{2}, \\
\zeta \in R_{c \delta}\left(\zeta^{\delta}\right) .
\end{array}
$$

From property (iii) in Theorem 5 , there is a small constant $a$, $0<a \leq c$ (independent of $\tau_{i}, i=1,2,3$ ), so that

$$
\phi(\zeta) \geq 2 \operatorname{Re} h(\zeta)+a \sum_{i=1}^{3} \tau_{i}^{-2}\left|\zeta_{i}-\tilde{\zeta}_{i}\right|^{2}, \quad \zeta \in R_{c \delta}\left(\zeta^{\delta}\right)
$$

where

$$
\begin{aligned}
h(\zeta)= & \sum_{i=1}^{3} \frac{\partial \phi}{\partial \zeta_{i}}\left(\zeta^{\delta}\right)\left(\zeta_{i}-\widetilde{\zeta}_{i}\right) \\
& +\frac{1}{2} \sum_{i, j=1}^{3} \frac{\partial^{2} \phi}{\partial \zeta_{i} \partial \zeta_{j}}\left(\zeta^{\delta}\right)\left(\zeta_{i}-\widetilde{\zeta}_{i}\right)\left(\zeta_{j}-\widetilde{\zeta}_{j}\right) .
\end{aligned}
$$

If we set $\tilde{a}=a^{3} / 3$, it follows, from (33), that

$$
\operatorname{Re} h(\zeta) \leq-\tilde{a}, \quad \zeta \in\{\zeta ; \phi(z) \leq \tilde{a}\} \cap \operatorname{supp} \bar{\partial} \psi_{d}
$$


In the sequel, we set $B_{e}=R_{e \delta}\left(\zeta^{\delta}\right)$ for each small $e>0$. For each $s \geq 0$, set

$$
\alpha_{s}=\bar{\partial}\left(\beta_{\delta} e^{s h}\right)=v_{\delta} e^{s h} \bar{\partial} \psi_{d}(\zeta):=\sum_{i=1}^{3} \alpha_{s, i} d \bar{\zeta}_{i} .
$$

Then, $\alpha_{s}$ is a $\bar{\partial}$-closed smooth $(0,1)$-form with $\operatorname{supp} \alpha_{s} \subset$ $R_{c \delta}\left(\zeta^{\delta}\right)=B_{c}$. Let $\chi$ be a smooth convex increasing function that satisfies $\chi(t)=0$ for $t \leq \tilde{a} / 2$ and $\chi^{\prime \prime}(t)>0$ for $t>\tilde{a} / 2$. Now, define

$$
\lambda_{s}(\zeta)=\phi(\zeta)+s^{2} \chi(\phi(\zeta)) .
$$

According to the weighted estimates of $\bar{\partial}$-equation on $\widetilde{D}$ (instead of $\widetilde{\Omega}$ ) and by using estimate (32) for each $s \geq 0$, there is $u_{s}$ which satisfies $\bar{\partial} u_{s}=\alpha_{s}$, and

$$
\left\|u_{s}\right\|_{\lambda_{s}} \leqslant \int_{\widetilde{D}-B_{c}}\left|\alpha_{s}\right|^{2} e^{-\lambda_{s}}+\int_{B_{c}} \sum_{i=1}^{3} \tau_{i}^{2}\left|\alpha_{s, i}\right|^{2} e^{-\lambda_{s}} d V .
$$

Since $\left|\alpha_{s, i}\right| \lesssim e^{s \operatorname{Re} h} \tau_{i}^{-1}$ and supp $\alpha_{s} \subset B_{c}$, it follows from (38) that

$$
\begin{aligned}
\int_{\widetilde{D}}\left|u_{s}\right|^{2} e^{-\lambda_{s}} d V & \lesssim \int_{B_{c}} \sum_{i=1}^{3} \tau_{i}^{2}\left|\alpha_{s, i}\right|^{2} e^{-\lambda_{s}} d V \\
& \lesssim \int_{\text {supp } \bar{\partial} \psi_{d}} e^{2 s \operatorname{Re} h-\phi-s^{2} \chi(\phi)} d V .
\end{aligned}
$$

We consider the integrand of the last integral. If $\phi(z) \geq \widetilde{a}$, then $\chi(\phi(z)) \geq \chi(\widetilde{a})>0$, so the $s^{2}$-term in the exponent predominates. On the other hand, if $z \in \operatorname{supp} \bar{\partial} \psi_{d}$ and $\phi(z) \leq$ $\widetilde{a}$, then (35) shows that the integrand tends to zero. Thus, for any $\epsilon_{0}>0$, there exist $s_{0}>0$ and a function $u_{s_{0}}$ so that $\bar{\partial} u_{s_{0}}=$ $\alpha_{s_{0}}$ and

$$
\int_{\widetilde{D}}\left|u_{s_{0}}\right|^{2} e^{-\lambda_{s_{0}}} d V \lesssim \int_{\text {supp } \bar{\partial} \psi_{d}} \epsilon_{0} d V \lesssim \epsilon_{0} \prod_{i=1}^{3} \tau_{i}^{2} .
$$

Since $\phi\left(\zeta^{\delta}\right)=0$, it follows, from the property (iii) of Theorem 5, that there is $e>0$, independent of $\zeta^{\delta}$, such that $\psi_{d}(z)=1$ and $\phi(z)<\tilde{a} / 2$ for all $z \in B_{e}$. Note that $\lambda_{s}$ is independent of $s$ for $z \in B_{e}$, and $u_{s_{0}}$ is holomorphic in $B_{e}$. By mean value theorem, we have

$$
\begin{array}{r}
\left|\frac{\partial u_{s_{0}}}{\partial \zeta_{k}}\left(\zeta^{\delta}\right)\right|^{2} \lesssim \tau_{k}^{-2} \prod_{i=1}^{3} \tau_{i}^{-2} \int_{B_{e}}\left|u_{s_{0}}\right|^{2} e^{-\lambda_{s_{0}}} d V \lesssim \epsilon_{0} \tau_{k}^{-2}, \\
k=1,2,3,
\end{array}
$$

and hence it follows that

$$
\left|Y u_{s_{0}}\left(\zeta^{\delta}\right)\right| \lesssim \sqrt{\epsilon_{0}} \max \left(\left|b_{k}\right| \tau_{k}^{-1}\right) .
$$

Now, set $f_{\delta}=\beta_{\delta} e^{s_{0} h}-u_{s_{0}}$. Then, $f_{\delta}$ is holomorphic on $\widetilde{D}=$ $\Phi_{\tilde{z}^{\delta}}^{-1}(D)$. Since $\beta_{\delta}\left(\zeta^{\delta}\right)=h\left(\zeta^{\delta}\right)=0$, it follows, from (30) and (42), that $f_{\delta}$ satisfies

$$
\left|Y f_{\delta}\left(\zeta^{\delta}\right)\right| \gtrsim \sum_{i=1}^{3}\left|b_{i}\right| \tau_{i}^{-1}
$$

provided $\epsilon_{0}$ is sufficiently small.
We want to show that $\sup _{\widetilde{\Omega}}\left|f_{\delta}\right| \leq C$, where $C>0$ is independent of $\delta$. Recall that $s_{0}>0$ is fixed. Thus, from the property (iii) of Theorem 5 , there is a uniform constant $C_{0}>$ 0 such that $\left|\beta_{\delta} e^{s_{0} h}\right| \leq C_{0}$. Let $r_{0}>0$ be the constant satisfying (28) and assume that $\zeta \in \widetilde{U}_{r_{0} / 2}(0)=\Phi_{\widetilde{z}^{\delta}}^{-1}\left(U_{r_{0} / 2}(0)\right)$. Since $f_{\delta}$ is holomorphic on $\widetilde{D}$, it follows, by (40) and mean value theorem, that there exists a constant $C_{1}>0$, independent of $\delta>0$, such that

$$
\left|f_{\delta}(\zeta)\right|^{2} \leqslant r_{0}^{-6} \int_{\widetilde{U}_{r_{0} / 2}(\zeta)}\left|f_{\delta}\right|^{2} d V \leq C_{1} .
$$

We need to show the boundedness of $f_{\delta}$ outside $\widetilde{U}_{r_{0} / 2}(0)$. Let $\chi_{1}$ and $\chi_{2}$ be smooth cutoff functions with

$$
\begin{array}{ll}
\text { (i) } \chi_{1}(z)=1 \quad \text { if }|z| \geq \frac{r_{0}}{2}, \quad \chi_{2}(z)=1 \quad \text { if } z \in \operatorname{supp} \chi_{1} \\
\text { (ii) } \chi_{2}(z)=0 & \text { if }|z| \leq \frac{r_{0}}{4},
\end{array}
$$

and set $\tilde{\chi}_{i}=\Phi_{\widetilde{z}^{\delta}}^{*}\left(\chi_{i}\right), i=1,2$. By Kohn's theorem on global regularity for the $\bar{\partial}$-equation, the following estimate for the solution of $\bar{\partial} u=\alpha$,

$$
\left\|\tilde{\chi}_{1} u_{s_{0}}\right\|_{4}^{2} \lesssim\left\|\tilde{\chi}_{2} \alpha_{s_{0}}\right\|_{4}^{2}+\left\|u_{s_{0}}\right\|^{2},
$$

holds on $D$ provided $s_{0}>0$ is sufficiently large. Note that $\tilde{\chi}_{2} \alpha_{s_{0}}=0$ because supp $\alpha_{s_{0}} \subset R_{c \delta}\left(\zeta^{\delta}\right) \subset \widetilde{U}_{r_{0} / 4}(0)$ for all sufficiently small $\delta>0$. Thus, we conclude from (40), (46), and the Sobolev lemma that

$$
\sup _{\widetilde{D}}\left|\widetilde{\chi}_{1} u_{s_{0}}\right| \lesssim\left\|\widetilde{\chi}_{1} u_{s_{0}}\right\|_{4}^{2} \lesssim\left\|u_{s_{0}}\right\|^{2} \leq C_{2},
$$

where $C_{2}$ is independent of $\delta$.

Combining (44) and (47) and by the fact that $\left|\beta_{\delta} e^{s_{0} h}\right| \leq$ $C_{0}$, we conclude that

$$
\sup _{\widetilde{D}}\left|f^{\delta}\right| \leq C
$$

where $C$ is independent of $\zeta^{\delta}$ and $\delta$. Therefore, it follows from (43) and (48) that

$$
C_{\widetilde{\Omega}}\left(\zeta^{\delta} ; Y\right) \geq C_{\widetilde{D}}\left(\zeta^{\delta} ; Y\right) \gtrsim \sum_{i=1}^{3}\left|b_{i}\right| \tau_{i}^{-1} .
$$

On the other hand, the polydisc $B_{c}=R_{c \delta}\left(\zeta^{\delta}\right)$ about $\zeta^{\delta}$ lies in $\widetilde{\Omega}$. So one obtains that

$$
C_{\widetilde{\Omega}}\left(\zeta^{\delta} ; Y\right) \leq C_{B_{c}}\left(\zeta^{\delta} ; Y\right)=\max \left\{\left|b_{k}\right|\left(c \tau_{k}\right)^{-1}: k=1,2,3\right\} .
$$

Thus, one concludes from (49) and (50) that

$$
C_{\widetilde{\Omega}}\left(\zeta^{\delta} ; Y\right) \approx \sum_{i=1}^{3}\left|b_{i}\right| \tau_{i}^{-1} .
$$

Set $L_{k}^{\prime}=\left(d \Phi_{\tilde{z}^{\delta}}^{-1}\right) L_{k}, k=1,2,3$, where $L_{k}$ 's are defined in (1) in terms of $z$-coordinates defined in Theorem 1. 
At $\zeta^{\delta}=\left(d \delta^{1 / \eta}, 0,-b \delta / d_{0}\left(\widetilde{z}^{\delta}\right)\right)$, from the holomorphic coordinate change of $\Phi_{\widetilde{z}^{\delta}}$ in Proposition 4 , we see that

$$
\begin{aligned}
L_{1}^{\prime} & =\frac{\partial}{\partial \zeta_{1}}+e_{1}\left(z^{\delta}\right) d_{0}\left(\widetilde{z}^{\delta}\right) \frac{\partial}{\partial \zeta_{3}}:=\frac{\partial}{\partial \zeta_{1}}+\widetilde{e}_{1}\left(z^{\delta}\right) \frac{\partial}{\partial \zeta_{3}} \\
L_{2}^{\prime} & =\frac{\partial}{\partial \zeta_{2}}+\left[d_{1}\left(\widetilde{z}^{\delta}\right)+e_{2}\left(z^{\delta}\right)\right] \frac{\partial}{\partial \zeta_{3}} \\
& :=\frac{\partial}{\partial \zeta_{2}}+\tilde{e}_{1}\left(z^{\delta}\right) \frac{\partial}{\partial \zeta_{3}}
\end{aligned}
$$

and that

$$
L_{3}^{\prime}=d_{0}\left(\widetilde{z}^{\delta}\right) \frac{\partial}{\partial \zeta_{3}}
$$

where $d_{0}\left(\widetilde{z}^{\delta}\right)=(1 / 2)\left(\left(\partial r / \partial z_{3}\right)\left(\widetilde{z}^{\delta}\right)\right)^{-1}$ and $d_{1}\left(\widetilde{z}^{\delta}\right)=-((\partial r /$ $\left.\left.\partial z_{3}\right)\left(\widetilde{z}^{\delta}\right)\right)^{-1}\left(\partial r / \partial z_{2}\right)\left(\widetilde{z}^{\delta}\right)$ and where $e_{i}=-\left(\partial r / \partial z_{3}\right)^{-1}\left(\partial r / \partial z_{i}\right)$, $i=1,2$. Since $\left(\partial r / \partial z_{i}\right)(0)=0, i=1,2$, and $\left|\partial r / \partial z_{3}\right| \approx 1$, independent of $\delta$, it follows that $\left|\widetilde{e}_{i}\right| \lesssim \delta, i=1,2$. Thus, if the vector $Y=\sum_{i=1}^{3} b_{i}\left(\partial / \partial \zeta_{i}\right)$ is written as $Y=\sum_{i=1}^{3} a_{i} L_{i}^{\prime}$, then it follows that

$$
\max \left(\left|b_{i}\right| \tau_{i}^{-1}\right) \approx \sum_{i=1}^{3}\left|a_{i}\right| \tau_{i}^{-1}
$$

Let us write $X=\sum_{i=1}^{3} a_{i} L_{i}$, and $Y=\left(\Phi_{\tilde{z}^{\delta}}^{-1}\right)_{*} X=$ $\sum_{i=1}^{3} a_{i} L_{i}^{\prime}=\sum_{i=1}^{3} b_{i}\left(\partial / \partial \zeta_{i}\right)$. From (51), (53), and the invariance property of the metric, it follows that

$$
C_{\Omega}\left(z^{\delta} ; X\right)=C_{\widetilde{\Omega}}\left(\zeta^{\delta} ; Y\right) \approx \sum_{i=1}^{3}\left|a_{i}\right| \tau_{i}^{-1}
$$

To obtain an upper bound for the Bergman metric, we note that $R_{c \delta}\left(\zeta^{\delta}\right) \subset \widetilde{\Omega}$. Thus, by elementary estimates, for any $f \in A^{2}(\widetilde{\Omega}):=L^{2}(\widetilde{\Omega}) \cap A(\widetilde{\Omega})$, we obtain that

$$
\left|\frac{\partial f}{\partial \zeta_{k}}\left(\zeta^{\delta}\right)\right|^{2} \lesssim \tau_{k}^{-2} \prod_{j=1}^{3} \tau_{j}^{-2}\|f\|_{L^{2}(\widetilde{\Omega})}^{2},
$$

for $k=1,2,3$. Therefore, it follows that

$$
b_{\widetilde{\Omega}}\left(\zeta^{\delta} ; Y\right) \lesssim\left(\sum_{k=1}^{3}\left|b_{k}\right| \tau_{k}^{-1}\right) \prod_{j=1}^{3} \tau_{j}^{-1},
$$

where

$$
\begin{aligned}
& b_{\widetilde{\Omega}}\left(\zeta^{\delta} ; Y\right) \\
& \quad=\sup \left\{\left|Y f\left(\zeta^{\delta}\right)\right|: f \in A^{2}(\widetilde{\Omega}), f(z)=0,\|f\|_{L^{2}(\widetilde{\Omega})} \leq 1\right\} .
\end{aligned}
$$

Combining (23) and (56), one concludes that

$$
B_{\widetilde{\Omega}}\left(\zeta^{\delta} ; Y\right)=\frac{b_{\widetilde{\Omega}}\left(\zeta^{\delta} ; Y\right)}{K_{\widetilde{\Omega}}\left(\zeta^{\delta}, \zeta^{\delta}\right)^{1 / 2}} \lesssim \sum_{k=1}^{3}\left|b_{k}\right| \tau_{k}^{-1} .
$$

To estimate the upper bound of the Kobayashi metric, set

$$
R=\min \left\{c \tau_{k}\left|b_{k}\right|^{-1}: k=1,2,3\right\} .
$$

Then,

$$
f(t)=\left(b_{1} t, b_{2} t,-\frac{b \delta}{2}+b_{3} t\right)
$$

defines a map $f: D_{R} \subset \mathbb{C} \rightarrow B_{c}=R_{c \delta}\left(\zeta^{\delta}\right) \subset \widetilde{\Omega}$ with $f_{*}\left(\left.(\partial / \partial t)\right|_{0}\right)=Y=\sum_{k=1}^{3} b_{k}\left(\partial / \partial \zeta_{k}\right)$. Hence,

$$
\begin{aligned}
K_{\widetilde{\Omega}}\left(\zeta^{\delta} ; Y\right) & \leq K_{B_{c}}\left(\zeta^{\delta} ; Y\right) \leq R^{-1} \\
& \leq \max \left\{\left|b_{k}\right|\left(c \tau_{k}\right)^{-1}: k=1,2,3\right\} \\
& \lesssim \sum_{k=1}^{3}\left|b_{k}\right| \tau_{k}^{-1} .
\end{aligned}
$$

Combining (51), (58), and (61), we obtain that

$$
C_{\widetilde{\Omega}}\left(\zeta^{\delta} ; Y\right) \approx B_{\widetilde{\Omega}}\left(\zeta^{\delta} ; Y\right) \approx K_{\widetilde{\Omega}}\left(\zeta^{\delta} ; Y\right) \approx \sum_{i=1}^{3}\left|b_{i}\right| \tau_{i}^{-1}
$$

and hence the invariance property implies that

$$
C_{\Omega}\left(z^{\delta} ; X\right) \approx B_{\Omega}\left(z^{\delta} ; X\right) \approx K_{\Omega}\left(z^{\delta} ; X\right) \approx \sum_{i=1}^{3}\left|a_{i}\right| \tau_{i}^{-1} .
$$

If we combine (3), (4), (22), and (63), a proof of Theorem 1 is completed.

\section{Conflict of Interests}

The authors declare that there is no conflict of interests regarding the publication of this paper.

\section{Acknowledgment}

This study was partially supported by Sogang University Research Grant of 2012.

\section{References}

[1] E. Bedford and J. E. Fornaess, "Biholomorphic maps of weakly pseudoconvex domains," Duke Mathematical Journal, vol. 45, no. 4, pp. 711-719, 1978.

[2] S. Cho, "A lower bound on the Kobayashi metric near a point of finite type in $\mathbb{C}^{n}$," The Journal of Geometric Analysis, vol. 2, no. 4, pp. 317-325, 1992.

[3] K. Diederich and J. E. Fornaess, "Proper holomorphic maps onto pseudoconvex domains with real-analytic boundary," Annals of Mathematics, vol. 110, no. 3, pp. 575-592, 1979.

[4] J. McNeal, "Lower bounds on the Bergman metric near a point of finite type," Annals of Mathematics, vol. 136, no. 2, pp. 339360, 1992.

[5] R. M. Range, "The Caratheodory metric and holomorphic maps on a class of weakly pseudoconvex domains," Pacific Journal of Mathematics, vol. 78, no. 1, pp. 173-189, 1978. 
[6] D. W. Catlin, "Estimates of invariant metrics on pseudoconvex domains of dimension two," Mathematische Zeitschrift, vol. 200, no. 3, pp. 429-466, 1989.

[7] S. Cho, "Estimates of invariant metrics on some pseudoconvex domains in $C^{n}$," Journal of the Korean Mathematical Society, vol. 32, no. 4, pp. 661-678, 1995.

[8] G. Herbort, "On the invariant differential metrics near pseudoconvex boundary points where the Levi form has corank one," Nagoya Mathematical Journal, vol. 130, pp. 25-54, 1993.

[9] S. Cho, "Estimates of invariant metrics on pseudoconvex domains with comparable Levi form," Journal of Mathematics of Kyoto University, vol. 42, no. 2, pp. 337-349, 2002.

[10] G. Herbort, "Invariant metrics and peak functions on pseudoconvex domains of homogeneous finite diagonal type," Mathematische Zeitschrift, vol. 209, no. 2, pp. 223-243, 1992.

[11] D. Catlin, "Boundary invariants of pseudoconvex domains," Annals of Mathematics, vol. 120, no. 3, pp. 529-586, 1984.

[12] J. Y. Yu, "Peak functions on weakly pseudoconvex domains," Indiana University Mathematics Journal, vol. 43, no. 4, pp. 12711295, 1994.

[13] Y. You, "Necessary conditions for Hölder regularity gain of $\bar{\partial}$ equation in $C^{3}$," submitted.

[14] S. Cho, "Extension of complex structures on weakly pseudoconvex compact complex manifolds with boundary," Mathematische Zeitschrift, vol. 211, no. 1, pp. 105-119, 1992.

[15] K. T. Hahn, "Inequality between the Bergman metric and Carathéodory differential metric," Proceedings of the American Mathematical Society, vol. 68, no. 2, pp. 193-194, 1978. 


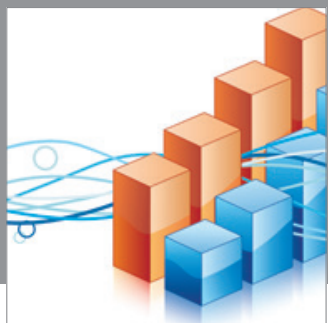

Advances in

Operations Research

mansans

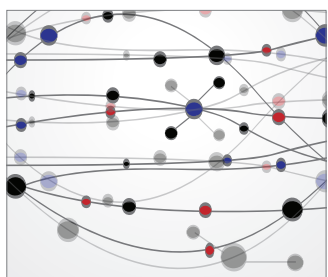

The Scientific World Journal
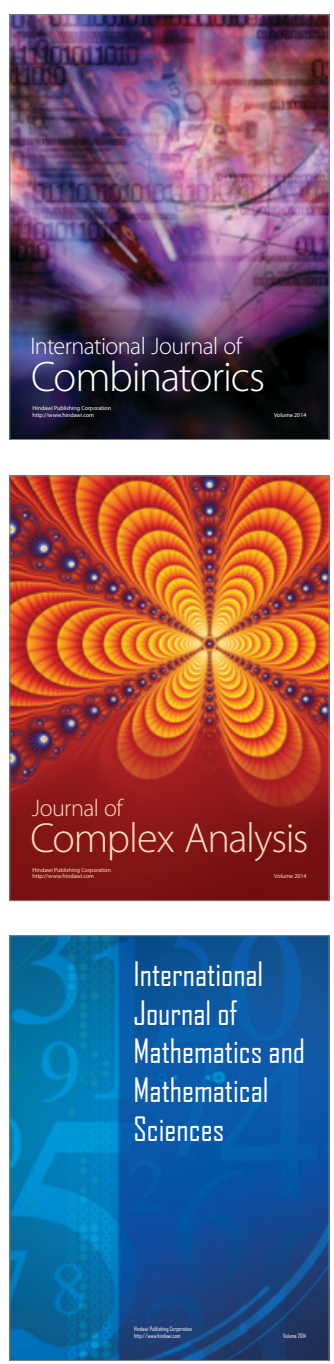
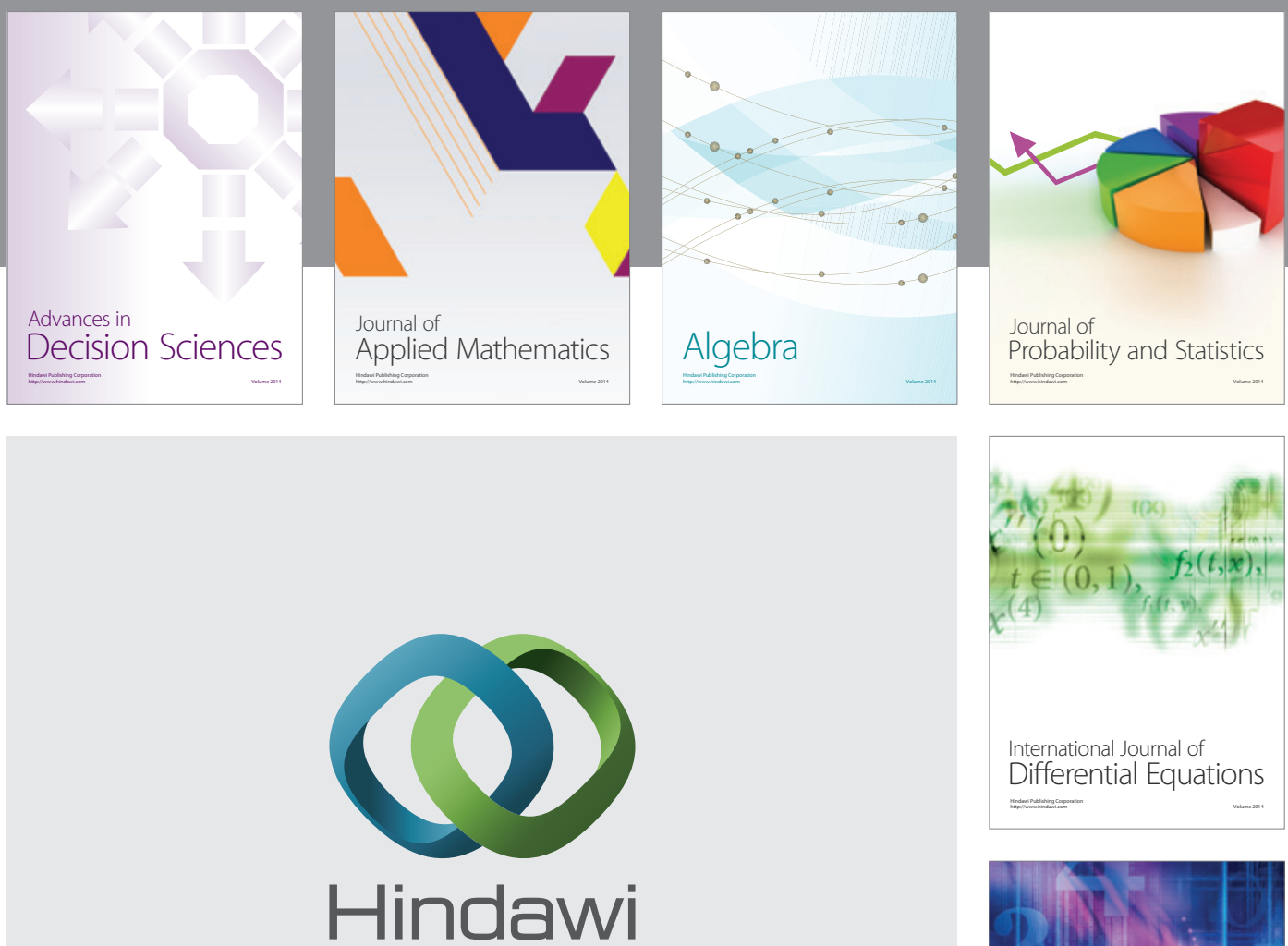

Submit your manuscripts at http://www.hindawi.com
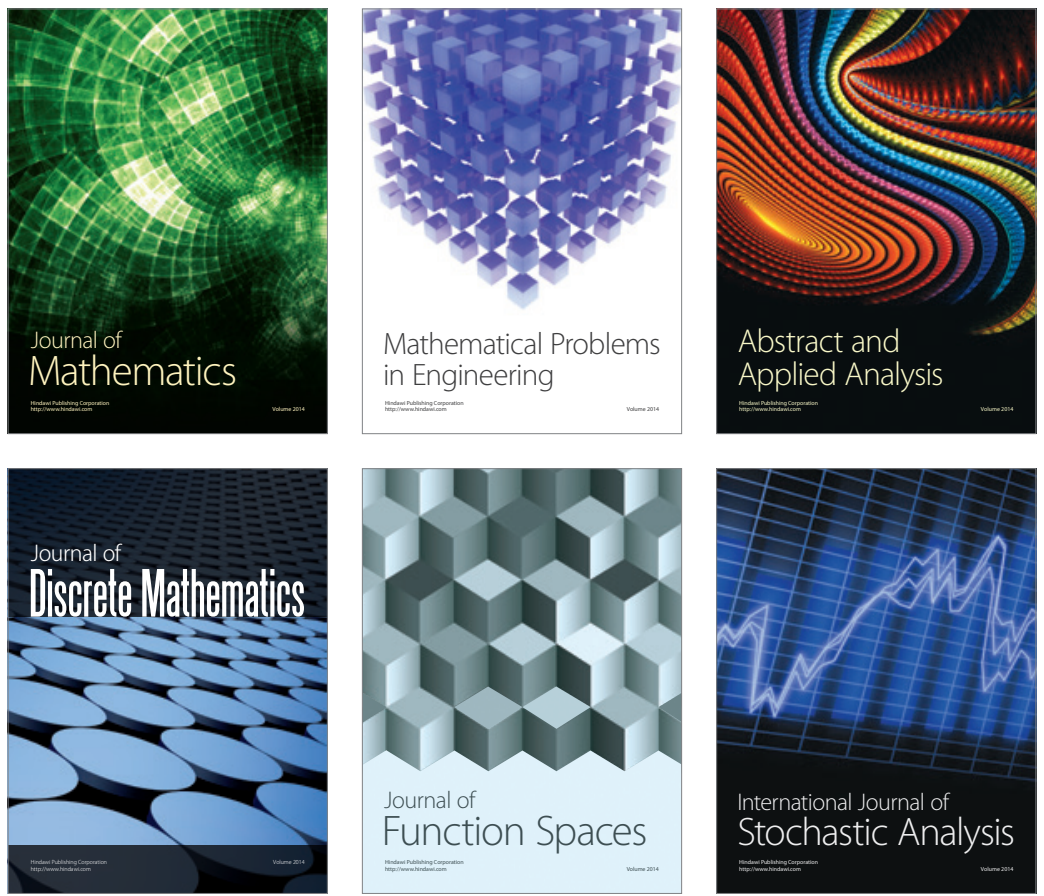

Journal of

Function Spaces

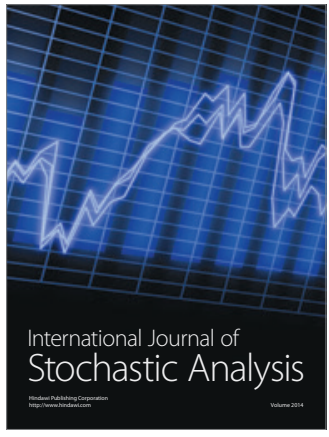

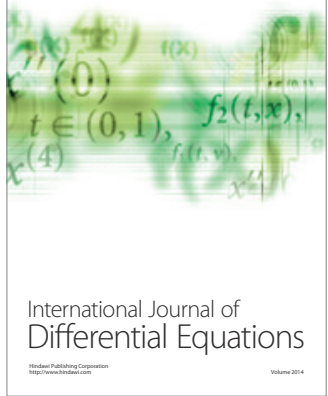
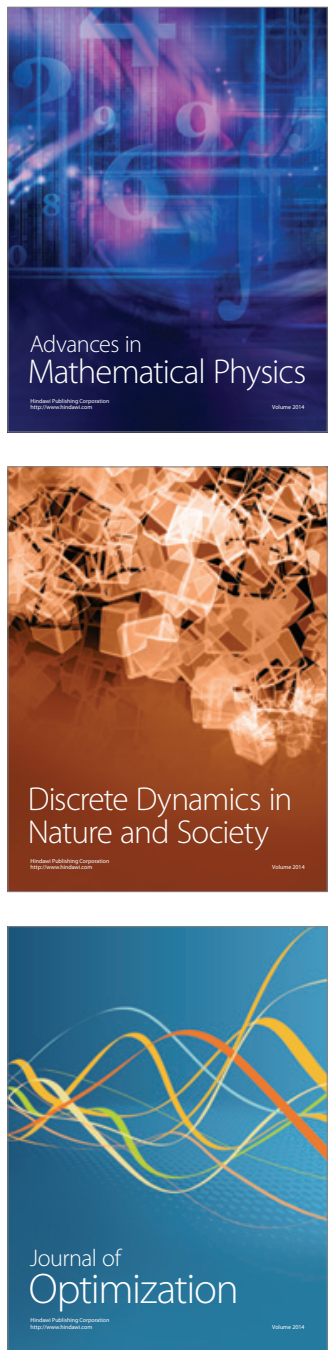